AEI-2005-091

gr-qc/0504100

\title{
Asymptotic Properties of Difference Equations for Isotropic Loop Quantum Cosmology
}

\author{
Martin Bojowald* and Adam Rej ${ }^{\dagger}$ \\ Max-Planck-Institut für Gravitationsphysik, Albert-Einstein-Institut, \\ Am Mühlenberg 1, D-14476 Golm, Germany \\ Division of Field Theory and Elementary Particle Physics, University of Silesia, Bankowa \\ 14,40-007 Katowice, Poland
}

\begin{abstract}
In loop quantum cosmology, a difference equation for the wave function describes the evolution of a universe model. This is different from the differential equations that arise in Wheeler-DeWitt quantizations, and some aspects of general properties of solutions can appear differently. Properties of particular interest are boundedness and the presence of small-scale oscillations. Continued fraction techniques are used to show in different matter models the presence of special initial conditions leading to bounded solutions, and an explicit expression for these initial values is derived.
\end{abstract}

\section{Introduction}

Quantum gravity can in general be expected to provide a foundation which is radically different from classical smooth background geometries in that space and time should be discrete. Evolution equations are then no longer given by differential equations in space and time, but by difference equations which in general can be approximated by differential equations only on large scales. This is realized explicitly in loop quantum cosmology 1, based on loop quantum gravity 2, 3, 4, where one obtains difference equations for the evolution of a universe [5]. This has physical implications, in particular concerning the singularity issue, but also conceptual ones since solutions of difference equations can generically behave differently from those of differential equations.

In this paper we focus on properties of solutions of isotropic loop quantum cosmology in the large volume region, where one expects differential equations to provide a good approximation [6, 7]. There are several cases where the boundedness of solutions is of

*e-mail address: mabo@aei.mpg.de

${ }^{\dagger}$ e-mail address: adamrej@box43.pl 
interest, for instance when a probability interpretation is used [8] or the physical inner product is studied [9]. From the continuum limit one can draw generic expectations, as in quantum mechanics, since one only needs to know whether regions of interest are classically

forbidden. In such a case, one expects exponential behavior of solutions of a differential equation and bounded solutions only in exceptional cases. However, since these issues can depend sensitively on precise values of a solution, a direct analysis of the difference equation without detour through the continuum limit is preferable.

This analysis is performed here with a general confirmation of the expectations. As a spin-off of the investigation we obtain a semi-analytical procedure which allows to determine initial values for bounded solutions. The resulting formula is much stronger than a numerical trial and error procedure, and does not only apply to those few models where exact solutions are known.

\section{Isotropic loop quantum cosmology}

Dynamical properties of a universe in isotropic loop quantum cosmology [10, 11, 12, are determined by a wave function $\psi_{\mu}$ subject to a difference equation of the type

$$
\begin{aligned}
& \left(V_{\mu+5}-V_{\mu+3}\right) e^{i k} \psi_{\mu+4}(\phi)-\left(2+k \gamma^{2}\right)\left(V_{\mu+1}-V_{\mu-1}\right) \psi_{\mu}(\phi) \\
& +\left(V_{\mu-3}-V_{\mu-5}\right) e^{-i k} \psi_{\mu-4}(\phi)=-\frac{4}{3} \pi G \gamma^{-3} \ell_{\mathrm{P}}^{2} \hat{H}_{\text {matter }}(\mu) \psi_{\mu}(\phi)
\end{aligned}
$$

in terms of volume eigenvalues $V_{\mu}=\left(\gamma \ell_{\mathrm{P}}^{2}|\mu| / 6\right)^{3 / 2}$ [13] which also show the geometrical meaning of the label $\mu \in \mathbb{R}$. A precise model is specified by the choice of a matter field $\phi$ and Hamiltonian $\hat{H}_{\text {matter }}(\mu)$ and by fixing $k$ to be zero for a flat model or one for a model of positive spatial curvature. The parameter $\gamma>0$ is the Barbero-Immirzi parameter of loop quantum gravity [14, 15, which fixes the scale of spatial discreteness. There are other versions of the equation, for instance obtained after a symmetrization procedure which changes the coefficients but not their large $\mu$ behavior. Our analysis will also apply to this equation. However, sometimes equations of higher order appear for which a similar analysis will be more complicated, as commented on later in this paper. Similarly, partial difference equations obtained for anisotropic models [16] require more care.

At small $\mu$ the difference equation can be used to understand quantum aspects of the classical singularity at $\mu=0$ [17, 18, 19, while at large $|\mu|$ one expects semiclassical behavior [6]. Here, we are interested in asymptotic properties of solutions at large $|\mu|$, in particular their boundedness or oscillatory properties. For simplicity, we base the analysis on phenomenological matter systems which do not require an additional matter field. In those cases, the equation takes the asymptotic form

$$
\hat{s}_{\mu+4}-\left(2+k \gamma^{2}\right) \hat{s}_{\mu}+\hat{s}_{\mu-4}=-\frac{8 \pi}{3} G \gamma^{2} a(\mu)^{-1} H(a(\mu)) \hat{s}_{\mu}
$$

with $\hat{s}_{\mu}:=e^{i k \mu / 4} \psi_{\mu}$ and $a(\mu)^{2}:=\gamma \ell_{\mathrm{P}}^{2}|\mu| / 6$. Matter Hamiltonians of interest are the cosmological constant term $H(a)=\Lambda a^{3}$, dust with $H(a)$ constant, radiation $H(a) \propto a^{-1}$, or 
a free massless scalar with plane wave initial conditions where $H(a) \propto a^{-3}$. In all these cases, the right hand side of (2) is of the form $-\Lambda|\mu|^{v}$ where $v$ is constant in a given model, but can in general take both signs. We simply denote constants characterizing the matter content by $\Lambda$, even if they do not correspond to a cosmological constant. Furthermore, without loss of generality we drop the constant $k$ and define $\gamma$ to be zero in flat models (after having absorbed it in $\Lambda$ on the right hand side). This brings us to the general equation

$$
\hat{s}_{\mu+4}-\left(2+\gamma^{2}\right) \hat{s}_{\mu}+\hat{s}_{\mu-4}=-\Lambda|\mu|^{v} \hat{s}_{\mu}
$$

to be studied.

Since the step size of the difference equation is four, defined for a wave function on the real line, values of $\psi_{\mu}$ between any $\mu$ and $\mu+4$ are unrelated by the equation. Depending on the initial conditions, there can thus be oscillations on arbitrarily small scales, which for intuitive reasons one would like to be suppressed in semiclassical regimes. If this is the case, a solution is called pre-classical 18, 17. This is partially a matter of initial conditions, but even within a given sequence at an integer lattice $\mu+4 \mathbb{Z}$ can small scale oscillations develop. Whether or not this can be suppressed depends now on the difference equation and thus has to be decided by an asymptotic analysis. Oscillations on larger scales are allowed and show the presence of a classically allowed regime. Also the occurrence of these oscillations can be seen by a general analysis.

In regimes where there are no large scale oscillations, which then are classically forbidden, one expects exponential behavior of solutions. Generically, the exponentially growing contribution will dominate such that the issue of boundedness of solutions arises in particular in models showing a classical recollapse 8 .

Both issues, pre-classicality and boundedness, have been used for an understanding of the semiclassical limit [8] and the physical inner product [9], even though the precise status of the conditions is not clear. Nevertheless, in those investigations one often needs to find special solutions, such as bounded ones, which are difficult to detect numerically in a reliable manner. It is thus necessary to have analytical tools available. For oscillations, generating function techniques have been used [20, 21], but this is applicable only in special cases (such as integer $v$ in our equation, and with ignoring the absolute value signs). The methods used here are more general and also apply to the boundedness issue.

It may happen that additional conditions will force $\mu$ to take discrete values [22] (enumerated by but not necessarily identical to integer numbers). It is therefore reasonable to construct tools in such a way that this possible discretization will be built in. To do so, we first notice that any real number can be represented as $\mu=n+\delta$ with $n \in \mathbb{Z}$ and $0 \leq \delta<1$, and define $s_{n}^{\delta} \equiv \hat{s}_{n+\delta}=\hat{s}_{\mu}$. Next, we find that the set of integer numbers splits into four disjoint subsets: $\mathbb{Z}=N_{1} \cup N_{2} \cup N_{3} \cup N_{4}$ where $N_{i}=4 \mathbb{Z}+i$. (We could have reduced solutions to only one subset by allowing $0 \leq \delta<4$. However, higher order equations, such as order four here, naturally arise in loop quantum cosmology, and so we leave this explicit for the sake of generality.)

When discretization is implied, the delta parameter is being fixed and the subset concept is very useful. If not, every subset gives rise to a channel $\hat{N}_{i}=N_{i}+\delta$ with $\delta \in[0,1)$. These 
four channels cover $\mathbb{R}$ completely. Equation (3) can then be rewritten:

$$
s_{n+4}^{\delta}-\left(2+\gamma^{2}\right) s_{n}^{\delta}+s_{n-4}^{\delta}=-\Lambda|n+\delta|^{v} s_{n}^{\delta} .
$$

For simplicity, we will drop the label $\delta$ in $s_{n}^{\delta}$ and simply write $s_{n}$. Different sequences will be distinguished from each other by the appearance of $\delta$ inside the absolute value in

$$
s_{n+4}-\left(2+\gamma^{2}\right) s_{n}+s_{n-4}=-\Lambda|n+\delta|^{v} s_{n} .
$$

\section{Cosmological constant}

We start with the case of a cosmological constant, i.e. $v=1$, in order to illustrate the main techniques. So equation (4) takes the form:

$$
s_{n+4}-\left(2+\gamma^{2}\right) s_{n}+s_{n-4}=-\Lambda|n+\delta| s_{n}
$$

\subsection{Generic asymptotic behavior}

We define

$$
\bar{s}_{n}=(-1)^{\frac{n-q}{4}} s_{n}
$$

where $n=4 m+q$ with $q$ the subset index $\left(q=i-1\right.$ for $\left.N_{i}\right)$ and $m$ an arbitrary integer. Introducing this into (5) results in

$$
\bar{s}_{n+4}+2 \bar{s}_{n}+\bar{s}_{n-4}=\Lambda|n+\delta| \bar{s}_{n}
$$

which is more convenient to investigate, starting with the $n \gg 1$ region.

To establish the asymptotic behavior we define

$$
\bar{s}_{n+4}=: h(n+4) \bar{s}_{n} .
$$

We have

$$
h(n+4)=\Lambda|n+\delta|-2-\frac{1}{h(n)}
$$

which allows expressing $h(n+4)$ in terms of $h(n)$.

For $n<0$, we can still keep $n$ and $\delta$ positive $\left(n \gg \Lambda^{-1}\right)$ but substitute $n \rightarrow-n$ and $\delta \rightarrow-\delta$ in (5). It is then more convenient to use $\bar{s}_{-(n+4)} / \bar{s}_{-n}$ instead, in order to investigate the regime of large negative $n$. This ratio is equal to

$$
\frac{\bar{s}_{-(n+4)}}{\bar{s}_{-n}}=\frac{1}{h(-n)}
$$

and the recurrence now is

$$
h(-n+4)+\frac{1}{h(-n)}=\Lambda|n+\delta|-2 .
$$




\subsubsection{Continued fraction}

With (7) and the analogous equation for $h(n), h(n)=\Lambda|n+\delta-4|-2-h(n-4)^{-1}$, we rewrite

$$
h(n+4)=\Lambda|n+\delta|-2-\frac{1}{\Lambda|n+\delta-4|-2-\frac{1}{h(n-4)}} .
$$

This can be iterated, for which it is convenient to use a suitable notation to indicate that the $k$-th step of iteration has been performed:

$$
\begin{aligned}
h_{0}(n+4) & =\Lambda|n+\delta|-2-\frac{1}{h(n)} \\
h_{1}(n+4)= & \Lambda|n+\delta|-2-\frac{1}{\Lambda|n+\delta-4|-2-\frac{1}{h(n-4)}} \\
h_{2}(n+4)= & \Lambda|n+\delta|-2-\frac{1}{\Lambda|n+\delta-4|-2-\frac{1}{\Lambda|n+\delta-8|-2-\frac{1}{h(n-8)}}} \\
& \vdots \\
h_{k}(n+4)= & \Lambda|n+\delta|-2-\frac{1}{\Lambda|n+\delta-4|-2-\frac{1}{\Lambda|n+\delta-8|-2-\cdots}} \\
& \\
&
\end{aligned}
$$

By construction,

$$
h_{k}(n+4)=h(n+4) \quad \text { for all } \quad k=0,1,2,3, \ldots
$$

As we observe, in the $k$-th step we have $k$ denominators with expressions as $\Lambda|\cdot|-2$ and an ending term $-h(n-4 k)^{-1}$. This process of iteration allows investigating the behavior of $h(n+4)$ in terms of arbitrary initial conditions of the form $s_{m_{0}}, s_{m_{0}+4}$.

For $n<0$ we perform the first step of iteration by

$$
\frac{1}{h(-n)}=\Lambda|n+\delta|-2-\frac{1}{\Lambda|n+\delta-4|-2-h(-n+8)}
$$

and have in the $k$-th step

$$
\begin{aligned}
& \frac{1}{h^{(k)}(-n)}=\Lambda|n+\delta|-2-\frac{1}{\Lambda|n+\delta-4|-2-\cdots} \\
& \cdots-\frac{1}{\Lambda|n+\delta-4 k|-2-h(-n+8(k+1))}
\end{aligned}
$$


with $k=1,2, \ldots$

\subsubsection{Convergence}

The iteration process leads easily to asymptotic relations (large $|n|$ behavior). In this section we will elucidate why this is the case, discussing only $n>0$ such that $n=4 m$, $m \gg 1$. Then the ending term for the $k$-th iteration is $-h(4(m-k))^{-1}$. Also for simplicity we set $k=m$, and thus the two last denominators of $h_{k}(n+4)$ are

$$
\frac{1}{\Lambda|n+\delta-4(k-1)|-2-\frac{1}{\Lambda|n+\delta-4 k|-2-\frac{1}{h(n-4 k)}}}=
$$

$$
\frac{1}{(4+\delta) \Lambda-2+\frac{1}{2-\Lambda \delta+\frac{1}{h(0)}}}
$$

To convince ourselves that choosing $h(0)$ arbitrarily does not greatly affect the large $n$ behavior of $h(n+4)$, we can simply reverse the whole procedure. We pick any value of $h(0)$ and, after a few steps, the system looses any information about it. To see this we present some examples. Because we always have inverse powers of $h(n)$, such as in

$$
h(4)=\Lambda \delta-2-\frac{1}{h(0)}
$$

and

$$
h(8)=(4+\delta) \Lambda-2-\frac{1}{h(4)},
$$

initial values for $h(0)$ such that $h(4) \sim 0$ (but not equal) could give rise to singular behavior. Indeed, $h(8)$ is large, but then

$$
h(12)=(8+\delta) \Lambda-2-\frac{1}{h(8)} \simeq(8+\delta) \Lambda-2 .
$$

So all information about the potentially dangerous value of $h(0)$ has been lost and the iteration proceeds as in the generic case.

For completeness, let us see now what happens when $h(4)$ is equal to zero. To proceed we introduce a parameter $\epsilon$ and a new initial value $h_{\epsilon}(0)$ such that

$$
\lim _{\epsilon \rightarrow 0} h_{\epsilon}(4)=\Lambda \delta-2-\frac{1}{h_{\epsilon}(0)}=0 \quad \text { and } \quad h_{\epsilon}(4) \neq 0 \text { for } \epsilon \neq 0 .
$$

As $\epsilon$ tends to zero, we will find $h(8)$ explode to infinity, and $h(12)$ tend to $8 \Lambda-2$. So this is just the limiting case of the previous one. 
There exists, however, one very special choice of $h(0)$ whose value is influential on the whole sequence $h(n)$. If we pick $h(0)$ such that the following sequence of an infinite number of constraints holds,

$$
\frac{1}{h(4 m)} \simeq(4 m+\delta) \Lambda-2 \quad \text { for all } m \in \mathbb{N},
$$

then cancellations occur within the continued fraction step after step such that the previously observed "forgetfulness" cannot be realized.

First of all, we can see that if the above sequence of constraints holds, it will hold only for one, unique initial value which we call $h_{\text {bounded }}(0)$ (unique for given $\Lambda, \delta$ and $k$ ). In App. $\mathrm{A}$ we present more discussion on this choice and prove that it leads to a vanishing solution for large $n$. The discussion above and in App. $\mathrm{A}$ signals that bounded solutions are permitted by our asymptotic relations. Furthermore, we will present tools, which allow us to find bounded solutions in a simple and explicit manner.

For values of $h(0)$ slightly different from $h_{\text {bounded }}(0)$, the iteration undergoes locally abrupt behavior, but after a few steps it smoothes again. So even for $h(0)$ very close to $h_{\text {bounded }}(0)$ the information about the initial value is being lost.

If $h(0)$ is different from $h_{\text {bounded }}(0)$ it is useful to split the fraction (8) into two pieces (recall that $k=m=\frac{n}{4}$ ). The lower part of the fraction, which is affected by initial value choices for which possibly the $\Lambda|\cdot|$ term does not dominate, will be called "part B". The upper part, unaffected by the initial value choice and for which $\Lambda|\cdot|-2$ is much bigger than one, will be called "part A". Thus, there is a $k_{\mathrm{B}}<k$ such that part B is identical to $h_{k_{\mathrm{B}}}(n+4)$, and part A is $h_{k-k_{\mathrm{B}}}(n+4)$ with part B used as initial condition.

From our arguments, we conclude that part $\mathrm{B}$ will become a small initial value contributing to part A. So what is left is part A, which is convergent but gives a negligible contribution, compared to the leading $\Lambda|n|$, as we move to the large $n$ regime. The same reasoning applies for any choice of an initial value $h(n-4 k)$. It is also easy to convince oneself that the above arguments are independent of our previous choice $n=4 \mathrm{~m}$.

\subsubsection{Generic growth}

If the initial value is different from $h_{\text {bounded }}$, there are at most finitely many near cancellations, and the following asymptotic relations (for large $n$ ) hold

$$
\begin{aligned}
& \frac{s_{n+4}}{s_{n}}=-h(n+4) \simeq 2-\Lambda|n| \\
& \frac{s_{-(n+4)}}{s_{-n}}=-\frac{1}{h(-n)} \simeq 2-\Lambda|n|
\end{aligned}
$$

since the fraction part of (8) or (91) can be ignored.

\subsection{Pre-classicality}

The previous results allow us to draw conclusions for the pre-classicality of solutions, in particular alternating behavior. 


\subsubsection{Subset splitting}

The subset splitting presented in Sec. 2 allows us to reduce

$$
\hat{s}_{n+4}-2 \hat{s}_{n}+\hat{s}_{n-4}=-\Lambda|n| \hat{s}_{n}
$$

to four equations

$$
s_{4(m+1)}-2 s_{4 m}+s_{4(m-1)}=-\Lambda|4 m+i+\delta| s_{4 m}
$$

each valid on one set $N_{i}(i=1,2,3,4)$. Each of them is of second order, and thus needs two initial values. There are no mixing terms, i.e. terms that mix $s_{n}$ from different $N_{i}$ subsets. So these four equations are fully independent.

Because for large $n$ (and for ratios different from $h_{\text {bounded }}$ )

$$
\frac{s_{n+4}}{s_{n}} \simeq 2-\Lambda|n|
$$

and analogously for $s_{-(n+4)} / s_{-n}$, we see that the asymptotic behavior excludes solutions where $s_{n}$ and $s_{n+4}$ (also $s_{-n}$ and $s_{-(n+4)}$ ) are of the same sign. This means that preclassicality within one subset $N_{i}$ is not preserved. Additionally, it may happen that the solution between different subsets is not pre-classical. Because, as we have seen, the original equation reduces to four independent equations of second order, for each equation we need two initial values. It may then happen, for example, that for $n \in N_{1} s_{n}$ oscillates with different phase than for $n \in N_{2}$.

Since we have just proved that generic solutions of (5) are not pre-classical within a subset, non-preclassicality between different subsets is only of secondary interest. However, we will meet equations which are pre-classical within a subset, and therefore it would be of interest to remove non-preclassical behavior between different subsets on the whole set $\mathbb{Z}=N_{1} \cup N_{2} \cup N_{3} \cup N_{4}$.

A simple example of such an equation is (6). The same reasoning as above implies now that $\bar{s}_{n+4}$ and $\bar{s}_{n}$ (also $\bar{s}_{-(n+4)}$ and $\bar{s}_{-n}$ ) are of the same sign, so the solutions are preclassical within a subset. We will show how to remove non-preclassical behavior among different subsets by a proper choice of initial values. In the next part we will present generalization to other models.

\subsubsection{Determining regimes leading to solutions of constant sign}

Again, we start with the positive $n$ direction of iteration which will simply generalize to the negative one. We rewrite (6) in a form

$$
\bar{s}_{n+4}=a(n) \bar{s}_{n}-\bar{s}_{n-4}
$$

where

$$
a(n)=\Lambda|n+\delta|-2
$$


Shifting the label by four and substituting $\bar{s}_{n}$ in the right hand side of (11), we get $\bar{s}_{n+4}$ in terms of $\bar{s}_{n-4}$ and $\bar{s}_{n-8}$. After $k$ iteration steps we reach

$$
\bar{s}_{n+4}^{(k)}=f^{(k)}(n) \bar{s}_{n-4 k}-g^{(k)}(n) \bar{s}_{n-4(k+1)}
$$

where $f$ and $g$ are functions of $n$ whose structure is of course $k$-dependent. For step $k+1$ we have

$$
\bar{s}_{n+4}^{(k+1)}=f^{(k+1)}(n) \bar{s}_{n-4(k+1)}-g^{(k+1)}(n) \bar{s}_{n-4(k+2)}
$$

and from (11)

$$
\bar{s}_{n-4 k}=a(n-4(k+1)) \bar{s}_{n-4(k+1)}-\bar{s}_{n-4(k+2)} .
$$

Substituting this in (12) we obtain

$$
\bar{s}_{n+4}^{(k+1)}=\left(a(n-4(k+1)) f^{(k)}(n)-g^{(k)}(n)\right) \bar{s}_{n-4(k+1)}-f^{(k)}(n) \bar{s}_{n-4(k+2)} .
$$

Comparing with previous expressions for $\bar{s}_{n+4}^{(k+1)}$ we obtain the recurrence relations

$$
\begin{aligned}
& f^{(k+1)}(n)=a(n-4(k+1)) f^{(k)}(n)-f^{(k-1)}(n) \\
& g^{(k+1)}(n)=f^{(k)}(n)
\end{aligned}
$$

having already used relation (14) in (13). To close these recurrence relations, we notice that

$$
f^{(0)}(n)=a(n) \quad \text { and } \quad g^{(0)}(n)=f^{(-1)}(n)=1 .
$$

We will now focus on relation (13), which has a symmetric form similar to (5). This suggests to introduce

$$
\rho(k+1, n)=\frac{f^{(k+1)}(n)}{f^{(k)}(n)}
$$

and rewrite equation (13) as

$$
\rho(k+1, n)=a(n-4(k+1))-\frac{1}{\rho(k, n)} .
$$

If we choose to use as initial values $\bar{s}_{4 m_{0}+q}$ and $\bar{s}_{4\left(m_{0}-1\right)+q}\left(q\right.$ is the subset constant for $N_{i}$, defined by $q=i-1$, and $m_{0}$ indicates the initial values in a given subset), we may write

$$
\bar{s}_{n+4}=f^{(\bar{k})}(n) \bar{s}_{4 m_{0}+q}-f^{(\bar{k}-1)}(n) \bar{s}_{4\left(m_{0}-1\right)+q}
$$

where $n-4 \bar{k}=4 m_{0}+q$ has been substituted by $\bar{k}=\frac{1}{4}\left(n-4 m_{0}-q\right)$. To assure that, for example, the solution is positive for large values of $n$, the condition $\bar{s}_{n+4}>0$ easily implies

$$
\operatorname{sgn}(f)\left(s_{4 m_{0}+q}-r(\bar{k}) s_{4\left(m_{0}-1\right)+q}\right)>0
$$

with

$$
r(\bar{k}) \equiv r\left(m_{0}, q\right)=\lim _{n \rightarrow \infty} \frac{1}{\rho(\bar{k}, n)}
$$


and $\operatorname{sgn}(f)$ is the sign of the $f$ coefficient which is discussed in App. B.1.

To find $r\left(m_{0}, q\right)$ it is sufficient to use equation (15). Arguments on convergence of (15) are given in App. B.2. So by a proper initial value choice we can make all four solutions on $\mathbb{Z}$ positive or negative. If $\mu$ is not discrete, one proceeds as before and makes all solutions positive or negative for all $\delta \in[0,1)$.

Equation (15) is easy to handle, but nevertheless it is instructive to discover some properties of this equation for a few particular cases (for simplicity putting $\delta=0$ ).

1. Subset $N_{1}, \bar{s}_{0}$ and $\bar{s}_{-4}$ as initial values:

Then $\bar{k}=\frac{n}{4}$ and shifting variables in (15) to

$$
\rho(k, n)=a(n-4 k)-\frac{1}{a(n-4(k-1))-\frac{1}{a(n-4(k-2))-\cdots}}
$$

we obtain

$$
\begin{aligned}
& \rho(n / 4, n)=-2-\frac{1}{4 \Lambda-2-\frac{1}{8 \Lambda-2-\cdots}} \\
& \cdots-\frac{1}{n \Lambda-2} .
\end{aligned}
$$

As expected, this expression is for large $n$ almost independent of $n$. The only parameter left is $\Lambda$, of which two values are particularly interesting:

a) $\Lambda=0$ : Because

$$
\frac{1}{-2-\frac{1}{-2-\cdots}}=-1
$$

we get

$$
\lim _{n \rightarrow \infty} \frac{1}{\rho(n / 4, n)}=-1
$$

in agreement with the results in App. C.3.

b) $\Lambda \gg 1$ : On the right hand side of Eq. (17) the fraction can then be neglected, and we have

$$
\lim _{n \rightarrow \infty} \frac{1}{\rho(n / 4, n)}=-\frac{1}{2} .
$$

This subset and these initial values are, however, very special because for $k=\bar{k}=\frac{n}{4}$ the $\Lambda$-term in $a(n-4 k)$ in (17) does not contribute. In other subsets, this does not happen. 
2. Subset $N_{2}, \bar{s}_{1}$ and $\bar{s}_{-3}$ as initial values:

This time, $n-4 \bar{k}=1$ such that $\bar{k}=\frac{n-1}{4}$ and

$$
\rho(\bar{k}, n)=\Lambda-2-\frac{1}{5 \Lambda-2-\frac{1}{9 \Lambda-2-\cdots}}
$$

We observe, as anticipated, the occurrence of $\Lambda$ at leading order. This follows from the subset splitting where for subset $N_{1}$ we have

$$
\bar{s}_{4(m+1)}+2 \bar{s}_{4 m}+\bar{s}_{4(m-1)}=\Lambda|4 m| \bar{s}_{4 m} .
$$

If we put $m=0$ in the above equation we see that the recurrence starts with $\bar{s}_{4}=-2 \bar{s}_{0}-\bar{s}_{-4}$ which is $\Lambda$-independent. For $N_{2}$, however, we have

$$
\bar{s}_{4(m+1)+1}+2 \bar{s}_{4 m+1}+\bar{s}_{4(m-1)+1}=\Lambda|4 m+1| \bar{s}_{4 m+1}
$$

and putting $m=0$ leads to $\bar{s}_{5}=(\Lambda-2) \bar{s}_{1}-\bar{s}_{-3}$. So $\Lambda$ appears at the zeroth iteration step. Now it is essential that due to (19) the regime for constant sign is sensitive to values of $\Lambda$ in contrast to the subset $N_{1}$. It is clear that this is the generic behavior, $N_{1}$ being the only exception.

For negative $n$ we proceed similarly, starting with $\bar{s}_{-(n+4)}^{(k)}=\bar{f}^{(k)}(n) \bar{s}_{-n+4 k}-\bar{g}^{(k)}(n) \bar{s}_{-n+4(k+1)}$ such that

$$
\begin{aligned}
& \bar{f}^{(k+1)}(n)=a(n-4(k+1)) \bar{f}^{(k)}(n)-\bar{f}^{(k-1)}(n) \\
& \bar{g}^{(k+1)}(n)=\bar{f}^{(k)}(n)
\end{aligned}
$$

allows us to write $\bar{s}_{-(n+4)}=\bar{f}^{(\bar{k}-1)}(n) \bar{s}_{4\left(m_{0}-1\right)+q}-\bar{f}^{(k)}(n) \bar{s}_{4 m_{0}+q}$ with $\bar{k}=\frac{1}{4}\left(n+4 m_{0}+q\right)$.

\subsection{Properties of initial values}

In this subsection we assume positive $n$; the generalization to negative $n$ is immediate.

\subsubsection{Asymptotic roles}

Using $\rho$ we write

$$
\bar{s}_{n+4}=f^{(\bar{k})}(n)\left(\bar{s}_{4 m_{0}+q}-\frac{1}{\rho(\bar{k}, n)} \bar{s}_{4\left(m_{0}-1\right)+q}\right)
$$

and, using the $n$-independence of $\rho$ for large $n$, clearly see the different roles played by the two initial values. The leading order contribution to $\rho$ for an arbitrary subset and arbitrary value of $m_{0}$ is

$$
\rho(\bar{k}, n) \simeq \Lambda\left|4 m_{0}+q+\delta\right|-2
$$

and thus it can happen that $\rho(\bar{k}, n)^{-1} \sim 0$ is a small number, depending on where initial conditions are stated $\left(m_{0}\right)$ in relation to $1 / \Lambda$. From Eq. (20) we conclude that the large $n$ behavior of $\bar{s}_{n+4}$ then depends very weakly on $\bar{s}_{4\left(m_{0}-1\right)+q}$. Thus, $\bar{s}_{4 m_{0}+q}$ dictates the rate of growth, which can easily be checked numerically as illustrated in Tab. 1. 


\begin{tabular}{c|cccc|cccc}
$\bar{s}_{5}$ & 0 & 1 & 2 & 10 & 5 & 5 & 5 & 5 \\
$\bar{s}_{9}$ & 5 & 5 & 5 & 5 & 0 & 1 & 2 & 10 \\
\hline $10^{-130} \bar{s}_{205}$ & 147 & 148 & 148 & 154 & 3.42 & 32.8 & 62.2 & 297
\end{tabular}

Table 1: Dependence on initial values in the subset $N_{2}$ with $\Lambda=5, \delta=0, m_{0}=2$.

\subsubsection{Bounded solutions}

From the asymptotic relation it follows that the solutions are generically growing fast and in general are not bounded. However, using the fact (App. B.2) that for $a(n)$ given in (111) the limit $r\left(m_{0}, q\right)$ exists we conclude that special solutions exist which are bounded. These are those solutions for which

$$
\lim _{n \rightarrow \infty}\left(\bar{s}_{4 m_{0}+q}-\frac{1}{\rho(\bar{k}, n)} \bar{s}_{4\left(m_{0}-1\right)+q}\right)=0
$$

i.e.

$$
\bar{s}_{4 m_{0}+q}=r\left(m_{0}, q\right) \bar{s}_{4\left(m_{0}-1\right)+q} .
$$

We easily see that $r\left(m_{0}, q\right)=h_{\text {bounded }}$. This shows that the infinitely many conditions (10) can indeed be satisfied and that the value $h_{\text {bounded }}$ exists. Most importantly, we now have a constructive procedure to compute the value for any given model via $r\left(m_{0}, q\right)$.

If one puts $\bar{s}_{4\left(m_{0}-1\right)+q}=0$ and compares this with (16) and the asymptotic relations one concludes that $f^{(\bar{k}(n))}(n)$ tends to (plus or minus) infinity and so is responsible for the asymptotic behavior. Putting $\bar{s}_{4 m_{0}+q}=0$ one finds that the same must hold for $f^{(\bar{k}(n)-1)}(n)$. Looking once again at (16) allows to understand why the solutions are bounded for $h_{\text {bounded }}$ and why this value is unique (for given $\Lambda, \delta$ and $m_{0}$ ). Only then is the growth of $\bar{s}_{4 m_{0}+q} f^{(\bar{k})}(n)$ canceled by the growth of $\bar{s}_{4\left(m_{0}-1\right)+q} f^{(\bar{k}-1)}(n)$.

\section{Arbitrary matter}

In this part, we investigate a general class of equations (44):

$$
s_{n+4}-b(n) s_{n}+s_{n-4}=-\Lambda|n+\delta|^{v} s_{n}
$$

with $b(n)=2+\gamma^{2}$, and real $v$. We must distinguish two cases.

\section{$4.1 \quad v>0$}

Here, the generalization from the discussion of a cosmological constant is straightforward. First, we introduce

$$
s_{n}=(-1)^{\frac{n-q}{4}} \bar{s}_{n}
$$


(where $q$ is the subset index). Equation (22) then has the form

$$
\bar{s}_{n+4}+b(n) \bar{s}_{n}+\bar{s}_{n-4}=\Lambda|n+\delta|^{v} \bar{s}_{n}
$$

We start with the positive $n$ direction of iteration and, to obtain the asymptotic behavior, define again

which, from (23), satisfies

$$
h(n+4)=\frac{\bar{s}_{n+4}}{\bar{s}_{n}}
$$

$$
h(n+4)=\Lambda|n+\delta|^{v}-2-\gamma^{2}-\frac{1}{h(n)} .
$$

For the negative $n$ direction of iteration we have

$$
\frac{\bar{s}_{-(n+4)}}{\bar{s}_{-n}}=\frac{1}{h(-n)}
$$

and

$$
h(-n+4)+\frac{1}{h(-n)}=\Lambda|n+\delta|^{v}-2-\gamma^{2} .
$$

The convergence arguments of the preceding section still apply, and we obtain

Proposition 1 Let $s_{n}$ be a solution to the difference equation (22) with $v>0$. If the ratio of initial values is different from $h_{\text {bounded, }}$ then the rate of growth is given by

$$
\frac{s_{n+4}}{s_{n}}=-\frac{\bar{s}_{n+4}}{\bar{s}_{n}}=-h(n+4)=2+\gamma^{2}-\Lambda|n|^{v}
$$

for large positive $n$, and by

$$
\frac{s_{-(n+4)}}{s_{-n}}=-\frac{\bar{s}_{-(n+4)}}{\bar{s}_{-n}}=2+\gamma^{2}-\Lambda|n|^{v}
$$

for large negative $n$.

So we conclude again that solutions within a subset are not pre-classical. Possible non-preclassicality among different subsets can be removed applying a generalized regime finding method, presented in Sec. 4.4, to each subset equation (23).

\section{$4.2 v<0$}

This model is very interesting physically, because it describes a universe with matter or radiation. As we will see, it contains many new features. Let us rewrite equation (22) in the form

$$
s_{n+4}-\left(2+\gamma^{2}-\Lambda|n+\delta|^{v}\right) s_{n}+s_{n-4}=0
$$

and define

$$
F(n)=2+\gamma^{2}-\Lambda|n+\delta|^{v}
$$

First, for negative $v$ we have $\lim _{n \rightarrow \pm \infty} F(n)=2+\gamma^{2}$ so we conclude that the results of Appendix $\mathrm{C}$ will be useful, and Eq. (45) there is indeed an asymptotic equation. 


\subsection{1 $\gamma \neq 0$}

We use $h(n+4)=s_{n+4} / s_{n}$ and reach

$$
h(n+4)=2+\gamma^{2}-\Lambda|n+\delta|^{v}-\frac{1}{2+\gamma^{2}-\Lambda|n+\delta-4|^{v}-\frac{1}{2+\gamma^{2}-\Lambda|n+\delta-8|^{v}-\cdots}}
$$

and

$$
\frac{1}{h(-n)}=2+\gamma^{2}-\Lambda|n+\delta|^{v}-\frac{1}{2+\gamma^{2}-\Lambda|n+\delta-4|^{v}-\frac{1}{2+\gamma^{2}-\Lambda|n+\delta-8|^{v}-\cdots}}
$$

In the large $n$ limit, using results of C.1.2, we have

Proposition 2 Let $s_{n}$ be a solution to the difference equation (22) with $v<0$ and $\gamma \neq 0$.

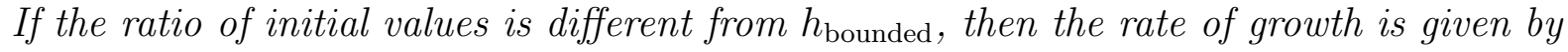

$$
\frac{s_{n+4}}{s_{n}}=h(n+4)=2+\gamma^{2}-x
$$

for large positive $n$, and by

$$
\frac{s_{-(n+4)}}{s_{-n}}=\frac{1}{h(-n)}=2+\gamma^{2}-x
$$

for large negative $n$, where

$$
x=\frac{2+\gamma^{2}-\sqrt{\gamma^{4}+4 \gamma^{2}}}{2}<1
$$

We conclude that solutions are preclassical within a subset, i.e. $s_{n+4}$ and $s_{n}$ (also $s_{-(n+4)}$ and $s_{-n}$ ) are of the same sign for all large $n$ (this means that also long distance oscillations are not possible). The only problem is to remove possible oscillating behavior between subsets, which is an easy task due to Sec. 4.4 (the limit of $\rho(\bar{k}, n)^{-1}$ will exist) and is achieved by picking up proper initial values.

However this does not exhaust all properties of the $\gamma \neq 0$ case. Long distance and short distance oscillations are excluded for large $n$, but they still may occur for small $n$ (the standard of smallness is parameter dependent).

If one picks $\Lambda$ and $\gamma$ in such a way that $F(n)>2$ for all $n$, then (with help of Appendix C) solutions are always non-oscillating. But for other choices one in general has $F(n)<2$ for $n \in\left[-n_{0}, n_{0}\right]$ and $F(n)>2$ for $n \in \mathbb{Z} \backslash\left[-n_{0}, n_{0}\right]$. As long as $F(n)<2$ the oscillating nature of solutions may manifest itself which, however, is not guaranteed: The condition $F(n)=2$ has solution $|\bar{n}|=\left(\gamma^{2} / \Lambda\right)^{1 / v}=\left(\Lambda / \gamma^{2}\right)^{1 /|v|}$. One may call $\bar{n}$ the value(s) of last oscillation. Depending on the phase of oscillation in a neighborhood of $\bar{n}$, the solution may lose its oscillating nature just before or just after $\bar{n}$. It is instructive to illustrate 


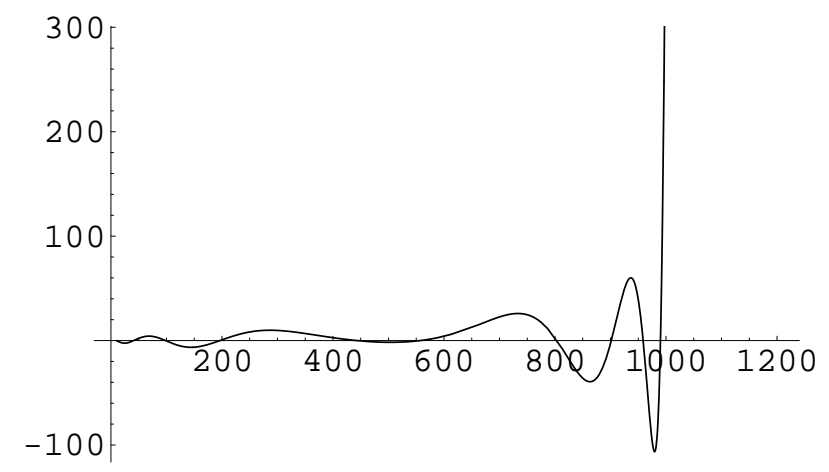

Figure 1: Typical behavior of a solution with last oscillation at $\bar{n}=1000$ and small $m_{0}$.

this feature with a numerical example: $N_{3}, \Lambda=5, \gamma=0.1, p=-1, s_{2}=2, s_{6}=3$. The solution passes zero for the last time at $n=474$ while the value of last oscillation is $\bar{n}=500$ (see also Fig. 11).

The necessary condition for small $n$ oscillations is now easy to state: The value of last oscillation must be greater than $4 m_{0}+q$ or smaller than $4\left(m_{0}-1\right)+q$, at least by a value of four. Otherwise, oscillations will not even have space to start. A sufficient condition will be formulated in what follows.

\subsection{2 $\gamma=0$}

This case is profoundly different because $F(n)<2$ for all $n$. Thus, with results in Appendix C we conclude that generic solutions are oscillating. We start investigations with the $v=-1$ case, moving to the large $n$ regime. For any $\epsilon>0$ we may always find such $N_{0}$ that for $N>N_{0}$ we have $2-\epsilon<F(n)<2$. We can use (50) in Appendix C] as an approximate solution, with one caveat. Since we are in the large $n$ regime, we cannot pick arbitrary $n_{0}$ in (50). Rather, it should be some value at which the large $n$ regime has already started. This means that, in general, we do not know anything about $s_{n_{0}}, s_{n_{0}-4}$, which are responsible for the amplitude of oscillation. However, the period of oscillation is independent of $s_{n_{0}}, s_{n_{0}-4}$, and we may use (50) to extract the period of oscillation. Using (49) and (50) we find the oscillating part of the solution for large $n$ to be given by $\sin (\omega n / 4)$ with period of oscillation $T=8 \pi / \omega$. It is more convenient to use half of the period, $\bar{T}:=T / 2=4 \pi / \omega$ which will be the distance between two subsequent zeros of oscillation. (Because $n$ is discrete, and its progress is four, it rarely happens that a solution takes zero value. Usually we have $s_{n}$ and $s_{n+4}$ of different sign. By zero of oscillation we mean then a number $z=n$ or $z=n+4$, depending on which absolute value is smaller, $s_{n}$ or $s_{n+4}$. $)$ With $\omega(a)=\tan ^{-1}\left(\sqrt{4-a^{2}} / a\right)$ and substituting $a$ by $2-\Lambda /|n|$ we reach an 


\begin{tabular}{c|cc|ccc|c}
$\Lambda$ & \multicolumn{2}{|c|}{ initial values } & $z_{1}$ & $z_{2}$ & $z_{2}-z_{1}$ & $\bar{T}(n)$ \\
\hline 1 & $s_{0}=2$ & $s_{4}=3$ & 332 & 604 & 272 & 271.83 \\
2 & $s_{1}=2$ & $s_{5}=3$ & 973 & 1269 & 296 & 297.48 \\
10 & $s_{10}=2$ & $s_{14}=5$ & 1806 & 1978 & 172 & 172.81
\end{tabular}

Table 2: Numerical compared to analytical values for the half-period (29).

approximate half period of oscillation of

$$
\bar{T}(n)=\frac{4 \pi}{\tan ^{-1}\left(\left(\sqrt{4 \Lambda}|n|-\Lambda^{2} /|n|^{2}\right) /(2-\Lambda /|n|)\right)} .
$$

With $\tan ^{-1}(x)=x-\frac{x^{3}}{3}+\left(x^{5}\right)$ for very large $n$ we can write

$$
\bar{T}(n) \sim \frac{4 \pi}{\sqrt{\Lambda}} \sqrt{|n|}
$$

Since (29) is indeed independent of $s_{n_{0}}$ and $s_{n_{0}-4}$, it should be a good approximation. To compare it with a numerical iteration we proceed as follows. Let $z_{1}$ and $z_{2}$ be two subsequent zeros of a solution. We define $\left(z_{1}+z_{2}\right) / 2=n$ and use this value in (29) to check if $\bar{T}(n) \simeq\left|z_{1}-z_{2}\right|$. This is confronted with a few numerical results in Tab. 2, showing indeed good agreement.

As already mentioned it is not possible to derive an exact value for the amplitude from (50). However, it is possible to derive the rate of growth which is proportional to $2\left(4 \Lambda /|n|-\Lambda^{2} /|n|^{2}\right)^{-1 / 2} \sim \sqrt{|n|}$.

Let us now drop the assumption $v=-1$. For large $n$ the oscillating part of the solution is $\sin (\omega(n) n)$ with

$$
\omega(n)=\frac{1}{4} \tan ^{-1}\left(\frac{\sqrt{4 \Lambda /|n|^{r}-\Lambda^{2} /|n|^{2 r}}}{2-\Lambda /|n|^{r}}\right)
$$

and $r=|v|$. One concludes that oscillations may be suppressed when $\lim _{n \rightarrow \infty} \omega(n) n=$ $\lim _{n \rightarrow \infty} n^{1-\frac{r}{2}}$ exists which implies $r \geq 2$. So for $\gamma=0$ we have

Proposition 3 For $-2<v<0$ the solutions are oscillating with increasing period $\frac{2 \pi}{\omega(n)}$. For $v \leq-2$ the phase is decreasing so rapidly that oscillations are suppressed. The regime finding method presented in Sec. 4.4 allows then to remove non-preclassical behavior among subsets.

The oscillating nature of solutions for $-2<v<0$ does not imply that they are not preclassical. The period of oscillation increases, and oscillations are of long distance type. For $v$ close to -2 , no oscillations occur for extremely large $n$. Fig. 2 illustrates the rate of growth of $\omega(n) n$ for $v=-1.9$.

Using the results of this subsection, it is now easy to state the sufficient condition for small $n$ oscillations in 4.2.1. The half period of oscillations for small $n$ should be smaller than the value $\bar{n}-4 m_{0}-q$ for the positive $n$ direction of iteration. The generalization to the negative $n$ direction of iteration is again straightforward. 


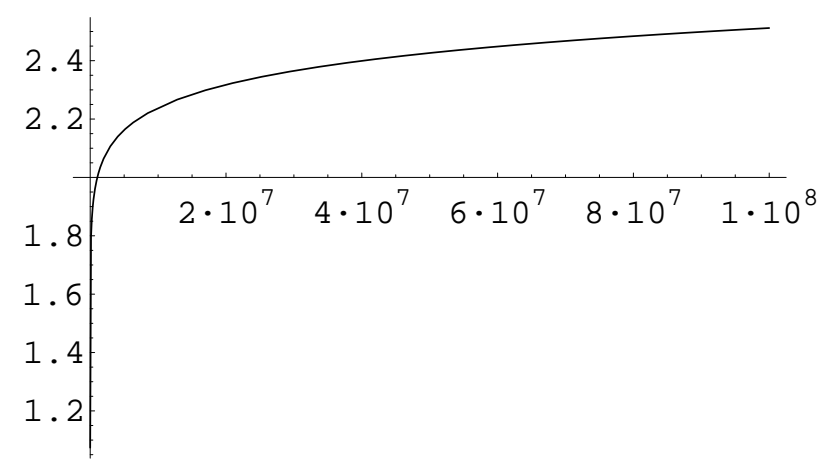

Figure 2: Phase $\omega(n) n$ of the approximate solution with $v=-1.9$.

\subsection{Generic asymptotic growth}

Solving the difference equation for $s_{n}$ given by the asymptotic behavior we obtain a growth of the form $\Lambda^{n / 4} \prod_{i=1}^{n / 4}|i|^{v}$ for $v>0$ from Prop. 1 and $c^{n}$ with a constant $c$ for $v<0$ and $\gamma \neq 0$ from Prop. [2. For $v<0$ and $\gamma=0$ solutions are oscillatory with growing amplitude at large $|n|$. We summarize this by

Theorem 1 (Generic asymptotic growth.) Let $\psi_{\mu}$ be a solution to the difference equation (1) without matter field. If $v>0$, then $\left|\psi_{\mu}\right|$ increases stronger than exponentially for $|n| \rightarrow \infty$. If $v<0$, then $\left|\psi_{\mu}\right|$ increases exponentially unless $\gamma=0$ in which case the increase in amplitude is given by a power law depending on $v$.

\subsection{Initial value representation}

So far, we discussed mainly asymptotic properties where the precise form of the coefficients in (11) is irrelevant. However, when we now turn to the initial value representation of bounded solutions, we must take into account that these initial values may be stated at small $n$ where the asymptotic form of the coefficients is not precise. In order to prove that the initial value representation is still valid we thus have to use the most general form of our difference equation

$$
a(n) s_{n+4}-b(n) s_{n}+c(n) s_{n-4}=f(n) s_{n}
$$

where $b(n), c(n), f(n)$ are arbitrary functions, and for $a(n)$ we only assume $a(n) \neq 0$ for all $n$ even though generalizing to the case where $a(n)$ has a finite number of zeros is not difficult.

Naturally, this equation again splits into four subsets $N_{1}, N_{2}, N_{3}, N_{4}$. We make an ansatz

$$
s_{n+4}^{(k)}=f^{(k)}(n) s_{n-4 k}-g^{(k)}(n) s_{n-4(k+1)}
$$


such that $s_{n+4}^{(k+1)}=f^{(k+1)}(n) s_{n-4(k+1)}-g^{(k+1)}(n) s_{n-4(k+2)}$. On the other hand, from (31)

$$
s_{n-4 k}=\frac{F(n-4(k+1))}{a(n-4(k+1))} s_{n-4(k+1)}-\frac{c(n-4(k+1))}{a(n-4(k+1))} s_{n-4(k+2)}
$$

with $F(n)=b(n)+f(n)$. Defining

$$
h(n-4(k+1))=\frac{F(n-4(k+1))}{a(n-4(k+1))}
$$

and

$$
j(n-4(k+1))=\frac{c(n-4(k+1))}{a(n-4(k+1))}
$$

and inserting (33) in (32) using above definitions, we reach

$$
\begin{gathered}
f^{(k+1)}(n)=h(n-4(k+1)) f^{(k)}(n)-g^{(k)}(n) \\
g^{(k+1)}(n)=j(n-4(k+1)) f^{(k)}(n)
\end{gathered}
$$

and thus

$$
f^{(k+1)}(n)=h(n-4(k+1)) f^{(k)}(n)-j(n-4 k) f^{(k-1)}(n) .
$$

Introduction of

$$
\rho(k, n)=\frac{f^{(k)}(n)}{f^{(k-1)}(n)}
$$

shows that the initial value representation is still valid, and has the form

$$
s_{n+4}=f^{(\bar{k})}(n)\left(s_{4 m_{0}+q}-\frac{j\left(4 m_{0}+q\right)}{\rho(\bar{k}, n)} s_{4\left(m_{0}-1\right)+q}\right)
$$

for the positive direction of iteration, the negative one following analogously. This representation is particularly important when the $\operatorname{limit}_{n \rightarrow \infty} \rho(\bar{k}(n), n)^{-1}$ exists. Violations of pre-classicality among different subsets may then be removed and bounded solutions exist. We have

Theorem 2 (Initial value representation of bounded solutions.) Let $a(n), b(n), c(n)$ and $f(n)$ be functions such that $\lim _{n \rightarrow \infty} \rho(\bar{k}(n), n)^{-1}=: r\left(m_{0}, q\right)$ exists, where $\rho(k, n)$ defined in (37) depends on the functions through (36) and (34), (35) with $F(n)=b(n)+f(n)$.

Then the difference equation (31), such as (1), in any subset $N_{q+1}$ has a solution bounded at positive $n$ whose initial values specified at $m_{0}$ satisfy $s_{4 m_{0}+q}=r\left(m_{0}, q\right) j\left(4 m_{0}+\right.$ q) $s_{4\left(m_{0}-1\right)+q}$. 


\section{Example: Euclidean cosmological constant model}

For a Euclidean cosmological constant model the equation

$$
s_{n+4}-2 s_{n}+s_{n-4}=\Lambda n s_{n}
$$

has been studied [9], which is here of interest because special solutions are known (note the absence of absolute values around $n$ in this toy model, which leads to explicitly known solutions). This can then be compared with our methods which easily apply to this equation, in particular the regime finding method and the condition for bounded solutions.

For positive $n \in N_{1}$, Eq. (39) has solution

$$
s_{n}=C_{1}^{N_{1}} J_{\frac{n}{4}+\frac{1}{2 \Lambda}}\left(\frac{1}{2 \Lambda}\right)+C_{2}^{N_{1}} Y_{\frac{n}{4}+\frac{1}{2 \Lambda}}\left(\frac{1}{2 \Lambda}\right) .
$$

For $n \rightarrow \infty$ the $Y$ Bessel function tends to minus infinity and the $J$ Bessel function to zero. Generically, the function $Y$ thus dominates, which agrees with our results for the generic asymptotic growth (Theorem 1): If $v=1$, we have $s_{n+4} \sim \Lambda n s_{n}$ from Prop. 1 such that $s_{n} \sim(4 \Lambda)^{n / 4}(n / 4-1)$ !. With Stirling's formula $n !=\sqrt{2 \pi n} n^{n} e^{-n}(1+O(n))$ we obtain $s_{n} \sim 2 \sqrt{2 \pi}(n-4)^{-1 / 2}(\Lambda(n-4))^{n / 4} e^{-n / 4+1}$. This can easily be seen to agree with the asymptotic growth of the Bessel function $Y_{\nu}(z) \sim-\sqrt{2 / \pi \nu}(e z / 2 \nu)^{-\nu}$ in the case $z=1 / 2 \Lambda, \nu=n / 4+z$ which determines the solution to the difference equation.

If we choose $s_{4 m_{0}}$ and $s_{4\left(m_{0}-1\right)}$ as initial values then $C_{2}^{N_{1}}$ is given by

$$
C_{2}^{N_{1}}=\frac{s_{4\left(m_{0}-1\right)} J_{m_{0}-1+\frac{1}{2 \Lambda}}}{\operatorname{det}\left(m_{0}, \Lambda\right)}\left(a_{1}-a\right)
$$

where $\operatorname{det}\left(m_{0}, \Lambda\right)=J_{m_{0}+\frac{1}{2 \Lambda}} Y_{m_{0}-1+\frac{1}{2 \Lambda}}-Y_{m_{0}+\frac{1}{2 \Lambda}} J_{m_{0}-1+\frac{1}{2 \Lambda}}$ and $a_{1}=J_{m_{0}+\frac{1}{2 \Lambda}} / J_{m_{0}-1+\frac{1}{2 \Lambda}}, a=$ $s_{4 m_{0}} / s_{4\left(m_{0}-1\right)}$. One can show that $\operatorname{det}\left(m_{0}, \Lambda\right)=\operatorname{det}(0, \Lambda)>0$ and $J_{m_{0}-1+\frac{1}{2 \Lambda}}\left(\frac{1}{2 \Lambda}\right)>0$ for all positive $\Lambda$ and $m_{0}$. So if for example $s_{4\left(m_{0}-1\right)+q}>0$, then for $a<a_{1}$ the solution for large $n$ is negative and for $a>a_{1}$ it is positive. For $a=a_{1}, C_{2}^{N_{1}}=0$ and the solution is bounded. In our previous notation (Theorem 2), this means $a_{1}=r\left(m_{0}, q\right)$ for the bounded solution.

Comparing the results, we obtain a continued fraction representation for the ratio of values of Bessel functions,

$$
\frac{J_{m_{0}-1+\frac{1}{2 \Lambda}}\left(\frac{1}{2 \Lambda}\right)}{J_{m_{0}+\frac{1}{2 \Lambda}}\left(\frac{1}{2 \Lambda}\right)}=2+4 \Lambda m_{0}-\frac{1}{2+4 \Lambda\left(m_{0}+1\right)-\frac{1}{2+4 \Lambda\left(m_{0}+2\right)-\cdots}}
$$

which in a similar form (with plus instead of minus signs in front of the fractions, which lead to modified Bessel functions of the first kind) can also be found in [23].

Generalization to other subsets and negative $n$ is easy to perform, and also confirms the presented method. 


\section{Conclusions}

The methods introduced here can be used to determine the generic growth of solutions to a difference equation of type (2). Moreover, even in generically unbounded cases there are analytical conditions for the presence and values of special initial conditions giving rise to bounded solutions. These conditions can be evaluated numerically in a constructive manner, without being sensitive to numerical instabilities which would hamper any trial and error approach. We have illustrated this in Sec. 5 in a model where explicit solutions to the difference equation are known.

Bounded solutions are often preferred, even though a clear reason is not known unless a physical inner product is used. One may impose boundedness as a final condition on allowed solutions, but usually in quantum cosmology initial conditions are imposed or arise at small volume. This, in general, does not guarantee that a wave function evolves to a bounded solution. In fact, bounded solutions are very special if there are classically forbidden regions at arbitrarily large volume. In such a case, growing solutions generically dominate. This is familiar from quantum mechanics, and has been shown here to be realized similarly at the level of difference equations directly. We emphasize, however, that the situation is more complicated with matter degrees of freedom, where the matter Hamiltonian is an operator and methods similar to those used here would require continued fractions of operators. Similarly, one has to deal with operator valued continued fractions in anisotropic models where now $a(n), b(n)$ and $c(n)$ as used in Sec. 4.4 are difference operators on labels other than $n$ [16, 24]. Higher order difference equations can also occur [10, 25], for which we can still introduce $h(n)$ as before. However, if there are also terms with $s_{n \pm 8}$, the equation for $h(n)$ obtained after dividing by $s_{n}$ contains $s_{n+8} / s_{n}=h(n+8) h(n+4)$ which makes it more complicated to solve.

There are two different origins of unboundedness of solutions of difference equations. The first appears in classically forbidden regions, and is not different from unboundedness in the continuum limit. This happens if $h(n)>1$, which can come from positive curvature $\gamma>0$ or negative matter terms such as a negative cosmological constant. There is also a second possibility, where now the behavior of solutions to a difference equation is completely different from that of a differential equation. This happens if $h(n)<-1$, which we have for a positive cosmological constant. In this case, the solution is not only unbounded but also has alternating behavior and is thus not pre-classical. Such a behavior cannot occur in the continuum limit, and expresses the presence of large curvature. It should be interpreted as a breakdown of the minisuperspace approximation where the integrated curvature is the only curvature quantity. Local curvature is small if the cosmological constant is small, but the integrated curvature $\Lambda|\mu|$ becomes large just from large volume. As the analysis here shows, this behavior is clearly distinguished and easily detectable. 


\section{Appendix}

\section{A The choice of $h_{\text {bounded }}$}

Let us assume that the sequence of constraints (10) holds. This means that if we take a large positive value of $n$, then there exists a small $\epsilon_{1}(n)$ such that

$$
h(n+4)=\Lambda|n|-2-\frac{1}{h(n)}=\epsilon_{1}(n)
$$

If we assume $h(n+4) \geq h(n)$ then $-h(n+4)^{-1} \geq-h(n)^{-1}$ and

$$
h(n+8)=\Lambda|n+4|-2-\frac{1}{h(n+4)} \geq\left(\Lambda|n|-2-\frac{1}{h(n)}\right)+4 \Lambda=4 \Lambda+\epsilon_{1}(n)
$$

implying in turn

$$
h(n+12)=\Lambda|n+8|-2-\frac{1}{h(n+8)} \geq \Lambda|n+8|-2-\frac{1}{4 \Lambda+\epsilon_{1}(n)} .
$$

A cancellation is then blocked by the $4 \Lambda$ term, which is in contradiction to the assumption of cancellations for all $n$. So we have $h(n+4)<h(n)$ and the sequence is decreasing. One can easily find out that $h(n)$ with the choice $h_{\text {bounded }}$ is much smaller than one but positive. Together with the previous prove this implies that solutions are vanishing asymptotically.

One can convince oneself, that this is the case by noting that the previous observations imply for large $n$ (and positive solutions) $0<s_{n}<c^{(n-m) / 4} s_{m}$ for some $m$ and $0<c<1$, and thus $\lim _{n \rightarrow \infty} s_{n}=0$. For negative solutions we have $0>s_{n}>c^{(n-m) / 4} s_{m}$ and again conclude $\lim _{n \rightarrow \infty} s_{n}=0$.

The generalization to large negative $n$ is straightforward.

\section{B Sign of $f$ coefficients and the existence of regimes}

\section{B.1 Sign of $f$ coefficients}

By definition, the coefficients $f$ obey the recurrence relation

$$
f^{(k+1)}(n)=a(n-4(k+1)) f^{(k)}(n)-f^{(k-1)}(n)
$$

with $f^{(0)}(n)=a(n)$ and $f^{(-1)}(n)=1$. We will prove that for $a(n) \geq 2$ for all $n$ all $f$ coefficients are positive. We have $f^{(1)}(n)=a(n-4) a(n)-1>a(n)=f^{0}(n)$ noting that

$$
a(n-4) a(n)-1 \geq 2 a(n)-1=a(n)+(a(n)-1)>a(n)
$$

because $a(n)-1 \geq 1>0$ as assumed. Let us assume now that $f^{(l)}(n)>f^{(l-1)}(n)$ for $l=0,1, \ldots, m$. Then

$$
f^{(m+1)}(n)=a(n-4(m+1)) f^{(m)}(n)-f^{(m-1)}(n) \geq 2 f^{(m)}(n)-f^{(m-1)}(n)>f^{(m)}(n)
$$


and by induction we finish the prove that $f^{(k)}(n)>f^{(k-1)}(n)$ for all $k$. So the sequence $f^{(k)}(n)$ is growing strictly monotonically and its initial values are positive, implying $f^{(k)}(n)>0$.

We now turn to the case where

$$
a(n)<2 \quad \text { for } n \in\left[n_{1}, n_{2}\right] \quad \text { and } \quad a(n) \geq 2 \quad \text { for } \mathbb{Z} \backslash\left[n_{1}, n_{2}\right]
$$

and consider the positive $n$ direction of iteration first. If we take large $n$ (fulfilling $n \gg n_{2}$ ), then $a(n-4 k) \geq 2$ for $k=0,1,2, \ldots, k_{0}$ where we have $f^{(k)}(n)>f^{(k-1)}(n)>0$ for $k=0,1,2, \ldots, k_{0}$, and so $\rho(k, n)>0$ for $k=0,1,2, \ldots, k_{0}$. We must then investigate what happens with the sign of $f$ for $k=k_{0}+1, \ldots, \bar{k}$ (one should note that $\bar{k}-k_{0}$ does not depend on $n$ ). With help of the already established formula

$$
\rho(k+1, n)=a(n-4(k+1))-\frac{1}{\rho(k, n)}
$$

and $\rho(0, n)=a(n)$ we find $\rho\left(k_{0}, n\right)$. We then use this formula again to see whether or not $\rho\left(k_{0}+1, n\right)=f^{\left(k_{0}+1\right)}(n) / f^{\left(k_{0}\right)}(n)$ changes its sign. If not, $f^{\left(k_{0}+1\right)}(n)$ is also positive. We then investigate $\rho\left(k_{0}+2, n\right)$, and so on. Every time $\rho(k, n)<0$ we have a change of sign, until we reach the desired value $\rho(\bar{k}, n)$. So one such iteration procedure allows to find both $\rho(\bar{k}, n)$ and $\operatorname{sgn}(f)=f^{(\bar{k})}(n) /\left|f^{(\bar{k})}(n)\right|$.

We will prove in the next subsection that for $a(n)$ as in (42) $\lim _{n \rightarrow \infty} \rho(\bar{k}(n), n)$ exists, and that $\rho(\bar{k}(n), n)$ is $n$-independent for large $n$, and so must equal $\rho\left(k_{0}(n), n\right)$. So it is sufficient to apply the above procedure for one value of large $n$.

\section{B.2 Existence of the regime constant $r\left(m_{0}, q\right)$}

The problem of existence of $r\left(m_{0}, q\right)=\lim _{n \rightarrow \infty} \rho(\bar{k}(n), n)^{-1}$ is closely related to the existence of continued fractions. We will show this for the positive $n$ direction of iteration. From Eq. (43) we have

$$
\rho(k, n)=a(n-4 k)-\frac{1}{a(n-4(k-1))-\frac{1}{a(n-4(k-2))-\cdots}} .
$$

Substituting $\bar{k}(n)=\frac{1}{4}\left(n-4 m_{0}-q\right)$ we reach

$$
\rho(\bar{k}(n), n)=a\left(4 m_{0}+q\right)-\frac{1}{a\left(4\left(m_{0}+1\right)+q\right)-\frac{1}{a\left(4\left(m_{0}+2\right)+q\right)-\cdots}} .
$$

So if $a(n)$ is of the form (42), there exists $l_{0}$ such that for all $l \geq l_{0}$ we have $a\left(4\left(m_{0}+l\right)+q\right) \geq$ 2. With help of results of C.2, we conclude that (44) is convergent, and for large $n$ almost $n$-independent. 


\section{The case $v=0$ as an asymptotic equation}

If $v \leq 0$, we are dealing with a difference equation whose coefficients are almost constant at large $n$ (or exactly constant for $v=0$ ) and which is of the form

$$
s_{n+4}-a s_{n}+s_{n-4}=0
$$

with a real parameter $a$. This equation can be analyzed by explicit solutions, but care must be taken in interpreting the results since the limiting value $a=2$ for large $n$ and $v<0$ is a critical value of the difference equation.

\section{C.1 Solutions}

We start with subset $N_{1}$ and introduce the generating function $F(x)=\sum_{m=m_{0}}^{\infty} s_{4 m} x^{m}$. From equation (45) we get $\left(x^{-1}-a+x\right) F(x)-s_{4 m_{0}} x^{m_{0}-1}+s_{4\left(m_{0}-1\right)} x^{m_{0}}=0$ and

$$
F(x)=\frac{x^{m_{0}} s_{4 m_{0}}-x^{m_{0}+1} s_{4\left(m_{0}-1\right)}}{1-a x+x^{2}} .
$$

To find solutions we must expand this generating function as a power series in $x$. One easily sees that the numerator will only result in a variable shift, and the main task is to expand

$$
G(x)=\frac{1}{1-a x+x^{2}}=\sum_{n=0}^{\infty} t_{n} x^{n} .
$$

We will use $1-a x+x^{2}=\left(x-x_{1}\right)\left(x-x_{2}\right)$ with roots

$$
x_{1}=\frac{a+\sqrt{a^{2}-4}}{2} \quad \text { and } \quad x_{2}=\frac{a-\sqrt{a^{2}-4}}{2}
$$

which are real if and only if $|a|>2$.

\section{C.1.1 $|a|>2$}

For this case, we write

$$
\frac{1}{\left(x-x_{1}\right)\left(x-x_{2}\right)}=\frac{1}{x_{1}-x_{2}}\left(\frac{1}{x-x_{1}}-\frac{1}{x-x_{2}}\right)
$$

and $\left(x-x_{1}\right)^{-1}=-x_{1}^{-1}\left(1-x / x_{1}\right)^{-1}=-x_{1}^{-1} \sum_{k=0}^{\infty}\left(x / x_{1}\right)^{k}$. From (46) we reach

$$
t_{n}=\frac{1}{x_{1}-x_{2}}\left(x_{2}^{-n-1}-x_{1}^{-n-1}\right)=\left(a^{2}-4\right)^{-1 / 2}\left(x_{2}^{-n-1}-x_{1}^{-n-1}\right) .
$$

For the large $m$ behavior we notice that for $a>2$ we have $x_{1}>1$ and $x_{2}<1$. For large $n$ the second term in bracket in (48) becomes negligible and the first one results in $\lim _{n \rightarrow \infty} t_{n}=\infty$. 


\section{C.1.2 $|a|<2$}

We make an analytic continuation to the principal sheet in (48) and prove that the $t_{n}$ remain real. We see that $x_{1}=\frac{1}{2}\left(a+i \sqrt{4-a^{2}}\right)=x_{2}^{*}$ and write $x_{1}=|r| e^{i \omega}, x_{2}=|r| e^{-i \omega}$. Before going further we notice $|r|=1$ so that the roots are pure phase. We rewrite equation (48) as

$$
t_{n}=-\frac{2}{\sqrt{4-a^{2}}} \sin (\omega(n+1))
$$

Thus, for $|a|<2$ solutions are oscillating with constant amplitude and $a=a_{0}=2$ is a critical point.

From $\omega=\tan ^{-1}\left(\sqrt{4-a^{2}} / a\right)$ we find the oscillation period $T=2 \pi / \omega$. Since $\lim _{a \rightarrow 2-} T(a)=$ $\infty$ the closer $a$ is to two, the longer the period is, and solutions slowly acquire the nonoscillating nature of $|a| \geq 2$ (in C.3 we show that also the case $a=a_{0}=2$ is non-oscillating).

Making the already mentioned variable shift in $F(x)$ we reach

$$
s_{4 m}=s_{4 m_{0}} t_{m-m_{0}}-s_{4\left(m_{0}-1\right)} t_{m-\left(m_{0}+1\right)}
$$

In terms of $n$ and for an arbitrary subset we have

$$
s_{n}=s_{n_{0}} t_{\frac{n-q}{4}-m_{0}}-s_{n_{0}-4} t_{\frac{n-q}{4}-\left(m_{0}+1\right)} \cdot
$$

We also conclude that if $a(n)$ is such a function that for all $\epsilon$ there is an $m_{0}$ for which $2-\epsilon<a(n)<2$ for all $n \geq m_{0}$ such that for large $n$ we have

$$
a(n+4) \simeq a(n) \simeq a(n-4)
$$

and (50) with $a \rightarrow a(n)$ is an approximate solution to $s_{n+4}-a(n) s_{n}+s_{n-4}=0$.

It should be noted that the bigger the value of $n$ is, the better condition (51) is fulfilled. However, it does not imply that $\omega(n)$, obtained after substituting $a$ by $a(n)$, is constant. Rather, it varies very slowly compared to the change of $s_{n}$ when $n$ is changed by four. One also must remember that $a=a_{0}=2$ is a critical point of the asymptotic equation, so substituting $a$ by two instead of observing condition (51) will not lead to correct results.

\section{C.2 Continued fractions}

We will employ another tool used to investigate loop quantum cosmology equations. This is a continued fraction, which we define by:

$$
\frac{1}{a-\frac{1}{a-\cdots}}=x
$$

Noticing $(a-x)^{-1}=x$ we have

$$
x^{2}-a x+1=0 .
$$


This is the denominator of $G(x)$ in (46) and so existence of the continued fraction is related to the problem considered before. For $|a|<2$, Eq. (52) has no solutions and so the continued fraction does not converge. To see from the previous context why this is the case we introduce $h(n+4)=s_{n+4} / s_{n}$ in (45) such that $h(n+4)=a-h(n)^{-1}$. Thus,

$$
\lim _{n \rightarrow \infty} h(n+4)=a-\frac{1}{a-\frac{1}{a-\cdots}}
$$

and one sees the close relationship between equation (45) and continued fractions. For $|a|<2$ solutions are oscillating, and so is $h(n+4)$, so the continued fraction does not exist. For $|a| \geq 2$, on the other hand, $h(n+4)=a-x$ and the continued fraction is well-defined.

In particular, if we assume $a \geq 2$ then $x=x_{2}$ from (47). For finite $n$ and if sufficiently many steps are taken,

$$
\frac{1}{a-\frac{1}{a-\frac{1}{\cdots-h_{0}}}}
$$

is almost $h_{0}$ independent, and so very close to $x$.

\section{C.3 The case $|a|=2$}

We start with the solution to (45) for $a=2, s_{n+4}-2 s_{n}+s_{n-4}=0$ which is

$$
s_{n+4}=(m+2) s_{n-4 m}-(m+1) s_{n-4(m+1)} .
$$

We pick $m$ in a such way that we have $s_{n+4}$ in terms of initial values at $n-4 m_{0}$ and $n-4\left(m_{0}+1\right)$, say: $s_{n+4}=\left(m_{0}+2\right) s_{n-4 m_{0}}-(m+1) s_{n-4\left(m_{0}+1\right)}$.

To obtain $s_{n+8}$ in terms of the initial values, we shift the label from $n$ to $n+4$ and choose $m$ such that $n+4-4 m=n-4 m_{0}$, i.e. $m=m_{0}+1$. Thus, $s_{n+8}=\left(m_{0}+3\right) s_{n-4 m_{0}}-$ $\left(m_{0}+2\right) s_{n-4\left(m_{0}+1\right)}$ implying

$$
s_{n+8}-s_{n+4}=s_{n-4 m_{0}}-s_{n-4\left(m_{0}+1\right)} .
$$

Because on the right hand side we have initial values, the progress of the sequence is constant. In the special case $s_{n-4 m_{0}}=s_{n-4\left(m_{0}+1\right)}$, the progress is zero and solutions are constant. So solutions in the case $a=a_{0}=2$ are in general linearly growing (except for one choice of initial values). This should be compared with the oscillating nature for $|a|<2$ and exponential growth for $|a|>2$. As we have already remarked, $a=a_{0}$ is a critical point of the asymptotic equation, resembling critical points of differential equations.

It is also interesting to discuss how the general procedure to find bounded solutions works here. For the subset $N_{1}$ we have $s_{n+4}=(n / 4+2) s_{0}-(n / 4+1) s_{-4}$ from which the condition

$$
s_{0}>\frac{n / 4+1}{n / 4+2} s_{-4}
$$


for a positive solution follows, and thus we must have $s_{0}>s_{-4}$ in the limit $n \rightarrow \infty$. Using our previous approach with the function $\rho$ and noting that

$$
\frac{1}{2-\frac{1}{2-\cdots}}=1
$$

we have $r\left(m_{0}, q\right)=\lim _{n \rightarrow \infty} \rho_{a=2}(\bar{k}(n), n)^{-1}=1$, reproducing $s_{0}>s_{-4}$.

The case $a=-2$ follows immediately after the substitution $s_{n}=(-1)^{(n-q) / 4} \bar{s}_{n}$.

\section{References}

[1] M. Bojowald and H. A. Morales-Técotl, Cosmological applications of loop quantum gravity, In Proceedings of the Fifth Mexican School (DGFM): The Early Universe and Observational Cosmology, pages 421-462. Lect. Notes Phys. 646, Springer-Verlag, 2004, gr-qc/0306008

[2] A. Ashtekar and J. Lewandowski, Background independent quantum gravity: A status report, Class. Quantum Grav. 21 (2004) R53-R152, gr-qc/0404018

[3] C. Rovelli, Quantum Gravity, Cambridge University Press, Cambridge, UK, 2004

[4] T. Thiemann, Introduction to Modern Canonical Quantum General Relativity, gr-qc/0110034

[5] M. Bojowald, Loop Quantum Cosmology IV: Discrete Time Evolution, Class. Quantum Grav. 18 (2001) 1071-1088, gr-qc/0008053

[6] M. Bojowald, The Semiclassical Limit of Loop Quantum Cosmology, Class. Quantum Grav. 18 (2001) L109-L116, gr-qc/0105113

[7] M. Bojowald and G. Date, Consistency conditions for fundamentally discrete theories, Class. Quantum Grav. 21 (2004) 121-143, gr-qc/0307083

[8] D. Green and W. Unruh, Difficulties with Closed Isotropic Loop Quantum Cosmology, Phys. Rev. D 70 (2004) 103502, gr-qc/0408074

[9] K. Noui, A. Perez, and K. Vandersloot, On the Physical Hilbert Space of Loop Quantum Cosmology, Phys. Rev. D 71 (2005) 044025, gr-qc/0411039

[10] M. Bojowald, Isotropic Loop Quantum Cosmology, Class. Quantum Grav. 19 (2002) 2717-2741, gr-qc/0202077

[11] M. Bojowald and K. Vandersloot, Loop quantum cosmology, boundary proposals, and inflation, Phys. Rev. D 67 (2003) 124023, gr-qc/0303072 
[12] A. Ashtekar, M. Bojowald, and J. Lewandowski, Mathematical structure of loop quantum cosmology, Adv. Theor. Math. Phys. 7 (2003) 233-268, gr-qc/0304074

[13] M. Bojowald, Loop Quantum Cosmology: II. Volume Operators, Class. Quantum Grav. 17 (2000) 1509-1526, gr-qc/9910104

[14] J. F. Barbero G., Real Ashtekar Variables for Lorentzian Signature Space-Times, Phys. Rev. D 51 (1995) 5507-5510, gr-qc/9410014

[15] G. Immirzi, Real and Complex Connections for Canonical Gravity, Class. Quantum Grav. 14 (1997) L177-L181

[16] M. Bojowald, Homogeneous loop quantum cosmology, Class. Quantum Grav. 20 (2003) 2595-2615, gr-qc/0303073

[17] M. Bojowald, Absence of a Singularity in Loop Quantum Cosmology, Phys. Rev. Lett. 86 (2001) 5227-5230, gr-qc/0102069

[18] M. Bojowald, Dynamical Initial Conditions in Quantum Cosmology, Phys. Rev. Lett. 87 (2001) 121301, gr-qc/0104072

[19] M. Bojowald, Initial Conditions for a Universe, Gen. Rel. Grav. 35 (2003) 1877-1883, gr-qc/0305069

[20] D. Cartin, G. Khanna, and M. Bojowald, Generating function techniques for loop quantum cosmology, Class. Quantum Grav. 21 (2004) 4495-4509, gr-qc/0405126

[21] D. Cartin and G. Khanna, Absence of pre-classical solutions in Bianchi I loop quantum cosmology, Phys. Rev. Lett. 94 (2005) 111302, gr-qc/0501016

[22] J. M. Velhinho, Comments on the kinematical structure of loop quantum cosmology, Class. Quantum Grav. 21 (2004) L109-L113, gr-qc/0406008

[23] R. Schroeppel, Item 99 in M. Beeler, R. W. Gosper and R. Schroeppel, HAKMEM (Cambridge, MA: MIT Artificial Intelligence Laboratory, Memo AIM-239, 1972), p. 36, http://www.inwap.com/pdp10/hbaker/hakmem/cf.html\#item99

[24] M. Bojowald, G. Date, and K. Vandersloot, Homogeneous loop quantum cosmology: The role of the spin connection, Class. Quantum Grav. 21 (2004) 1253-1278, gr-qc/0311004

[25] K. Vandersloot, On the Hamiltonian Constraint of Loop Quantum Cosmology, gr-qc/0502082 